\title{
Incidence of iatrogenic malnutrition in patients on enteral nutrition in the University of Nigeria Teaching Hospital
}

\author{
Osinachi Imaabasi Iwuoha ${ }^{1 *} \&$ Dr Christie Divine Akwaowo ${ }^{2}$ \\ ${ }^{I}$ Department of Human Nutrition and Dietetics, Michael Okpara University of Agriculture, Umudike, Abia \\ State, Nigeria \\ ${ }^{2}$ Community HealthDepartment, University of Uyo Teaching Hospital, Uyo, Akwa Ibom State, Nigeria
}

\begin{abstract}
The study assessed the incidence of iatrogenic malnutrition (also known as physician-induced or hospital induced malnutrition) in patients on enteral nutrition in the University of Nigeria Teaching Hospital Ituku-Ozalla, Enugu. A sample of twenty patients $(60 \%$ male and $40 \%$ female) was studied.The result showed that the elderly (55\%) and children(15\%) were the most affected. Assessments revealed that about $35 \%$ of the patients were malnourished. The causes of malnutrition included non-compliance to feeding time, delay in referring patients to the dieticians by physicians and underlying nutritional related diseases such as dysphagia(35\%), renal diseases (15\%) and sepsis(15\%). Effects of malnutrition in patients included paleness, anaemia, oedema, wasting, susceptibility to diseases, and in severe cases death. Recommendations have been made to reduce the incidence and prevent the harsh effects of malnutrition in hospitalized patients.
\end{abstract}

Keywords:Iatrogenic malnutrition; Clinical nutrition; Dieticians; Hospitalised patients; Enteral nutrition

\section{Introduction}

The problem of malnutrition in hospitalized patients was revealed in a 1974 article "The skeleton in the hospital's closet". Today malnutrition in hospitals remains a serious issue. According to the World Health Organization (WHO), malnutrition is the gravest single threat to global public health. Malnutrition maybe an excess of important nutrients in the body or it may also refer to their lack thereof. Usually, causes of malnutrition include poor eating habits with either too much or too little intake of the essential nutrients needed by the body. But not all forms of malnutrition can be attributed to bad eating habits or limited access to food; some may have iatrogenic effect or a result of iatrogenesis.

Iatrogenesis or iatrogenic effect derives from a Greek term meaning "brought forth by the healer" or "physician-produced" (Jacobs, Benavidez, Bacha, Walters\& Jacobs, 2008). Iatrogenesis thus describes any medical condition, disease or adverse event resulting from medical treatment (British Medical Association Illustrated Medical Dictionary, 2008). Malnutrition on the other hand describes a state in which a prolonged lack of one or more nutrient retards physical development or causes specific clinical disorders e.g. iron deficiency anaemia, goitre, and so on. Malnutrition is also defined as an impairment of health resulting from a deficiency, excess or imbalance of nutrients. It includes under nutrition and over nutrition (Shubhangini, 2002).

Iatrogenic malnutrition could therefore be defined as malnutrition due to various medications or some complications to medical treatment or procedure. It may be caused by negligence among medical personnel including doctors, nurses, therapists, or caregivers who are attending to a particular patient. Or it could just be an unfortunate side effect of some forms of medication or treatment procedure.The Oxford Concise Medical Dictionary (2002) defines iatrogenic malnutrition as malnutrition that occurs as a result of medical conditions, disease or adverse events resulting from medical treatment. It is a deficiency in energy, protein or other specific foods that produces measurable changes in body function. It is a condition that results from treatment as either an unforeseen or inevitable side effect.

Today, malnutrition in hospitals remains a serious issue and affects patients of all ages-from infants in the neonatal intensive care unit (ICU) to geriatric patients (Grove et al, 2008). Many patients reach the hospital in a malnourished state and there malnutrition is worsened by hospitalization while others are admitted into hospital with satisfactory nutritional state but become malnourished during their stay in the hospital (Aboaja, 2010). The link between malnutrition on admission to hospital and risk of it increasing during hospital stay is now well established in all parts of the world where studies have been conducted. Malnutrition among hospitalized patients interferes with recovery thereby increasing hospital stay and health care cost.

Furthermore, the disease itself and its associated complications indicate loss of muscle tissue, which requires long period of rehabilitation. Although nutrition support can partially ameliorate these changes, it is very often either inadequate or not given at all and leads to the so-called iatrogenic malnutrition (Aboaja, 2010). 
Incidence of iatrogenic malnutrition in patients on enteral nutrition in the University of....

\subsection{Statement of Problem}

Despite the realization of hospital malnutrition more than 30 years ago, it remains a significant problem. Many illnesses or injuries for which patients are admitted predispose them to malnutrition. Also prolonged hospital stay in itself, predispose patients to malnutrition. (Singhet al, 2006).Malnutrition during hospital admission is a risk factor for an unfavourable outcome. Prolonged hospital stay delays recovery and increases cost. A significant proportion of patients have sub-optimal nutritional intake compared with their needs during hospitalization (Kondrupet al, 2002). The reason for sub-optimal intake could be due to drug effects on nutrients. Most times drugs can affect appetite, altering food intake. Drugs may increase or decrease appetite, causing nausea, constipation and/or dry mouth, thereby altering nutrient absorption. Some drugs increase gastro-intestinal(GI) motility, decreasing food absorption. The problem for this study therefore was to examine the incidence of iatrogenic malnutrition on patients on enteral nutrition in the University of Nigeria Teaching Hospital Ituku-Ozalla, Enugu State.

\subsection{Specific objectives}

The specific objectives of the study therefore included:

1. To determine the incidence of iatrogenic malnutrition in patients on enteral feeds

2. To ascertain promptness in commencing enteral feeds among hospitalized Patients in UNTH

3. To evaluate the adequacy of feeding in patients on enteral feeds in UNTH

4. To suggest possible ways of combating iatrogenic malnutrition among hospitalized patients

\subsection{Significance of the study}

It is hoped that this study would re-emphasize the need for attention to be given to patients' nutritional status. If possible Dietitians should be invited to assess the nutritional status of patients and develop nutritional care plan for patients. This is to prevent malnutrition while on admission. This study also aims at creating awareness on the importance of attaching dietitians to each patient once admitted.

\subsection{Incidence of Iatrogenic Malnutrition}

\section{Review of Related Literature}

Malnutrition occurs during hospitalization after patients have been fed inadequate diets for several weeks. However, estimating the prevalence of hospital - induced malnutrition is difficult because of difficulties involved in recording accurately patients' nutritional status. Over the past 34 years, researchers have used different criteria to define hospital malnutrition, and studies have been conducted worldwide on populations that differ in socio-economic status, educational level, age group and severity of illness. Study group size also varies. A study reported in the 1970 s suggested that $30 \%$ to $50 \%$ of hospitalized people left the hospital in worse nutritional condition than when they arrived as patients. Hospitals generally have improved their menus since then, though hospitalization still does not guarantee optimal nutrition. A study carried out in 1996 on 57 hospitals found that only four hospital routinely offered patients menus that met all federal dietary guidelines, fewer than $20 \%$ kept cholesterol below $300 \mathrm{mg}$ /day, while half kept salt intake below $6 \mathrm{~g} /$ day and dietary fibre at more than 20g/day(Robert, 1997). Based on many report worldwide, an estimated $13 \%$ to $69 \%$ of hospitalized patients are malnourished (Weinsieret al, 2008). Malnutrition affects patients of all ages - from infants in the neonatal intensive care unit to geriatric (older patients) (Bristrainet al, 1996). It has been associated with an increased length of hospital stay, increased morbidity and mortality, impaired respiratory and cardiac function, decreased immune function and poor growth in infants and children (Correiaet al, 2005).

Results of a research carried out by Agabain 2006 among 76 patients (on dialysis) with chronic renal failure and 48 controls in Jos University Teaching Hospital, Nigeria, revealed that the mean body mass index was significantly low in the patients compared to the control. Underweight malnutrition was present in $21.6 \%$ of the patients compared with one of the control. Protein Malnutrition was also present in $3.2 \%$ of patients with chronic renal failure and $21 \%$ of the control subjects. In Maiduguri, Borno State of Nigeria, 120 children, 70 males and 50 females between the ages of 6-24 months at the paediatric wards of the University of Maiduguri Teaching Hospital, Maiduguri, Nigeria, laboratory findings showed all the children have one or more form of infections with Protein-Energy Malnutrition (PEM). Up to $41.6 \%$ had gastroenteritis, 21.7\% had malaria, $6.6 \%$ had measles, 5.0\% had pulmonary TB and 40\% had iron deficiency anaemia. Marasmus was highest in both male and female, $34.3 \%$ and $36 \%$ respectively for males and females between the ages of 6-12 months while kwashiorkor was highest among children between the ages of 13-18 months in both males and females. (Hamidu et al, 2003). Another research carried out by Hamidu in 2003 in Maiduguri among 120 patients showed a relationship between dietary patterns of malnourished children on hospital admission to the socio-economic status and education level of parents of children at the paediatrics wards of the University of Maiduguri Teaching Hospital and the State Specialist Hospital Maiduguri, Nigeria. The result of the research showed that $80 \%$ of the mothers of the malnourished children had no formal education, $18.3 \%$ attended primary school, 
$1.7 \%$ Islamic education. This indicates that the dietary pattern, socio-economic status and level of education of mothers predispose their children more to malnutrition while on hospital admission.

In the United Kingdom, malnutrition risks have been identified in $20 \%$ to $60 \%$ of hospital admission to medical, surgical, elderly and orthopaedic wards. A study of 550 consecutive hospital admission determined malnutrition in $40 \%$ of patients. Further hospitalization with surgery or other medical treatments often result in additional weight loss. Although malnutrition is common in hospital admission, it has been reported as undiagnosed in up to $70 \%$ cases (Peakeet al, 2005). Kelllyet al (2000) assigned likelihood of malnutrition scores based on serum folate and vitamin $\mathrm{C}$ level, anthropometric measurements, lymphocyte count, and hematocyte levels in 134 patients at a Teaching Hospital. Kelllyet al(2000) also found that among 219 patients in an academic inner city hospital in the United Kingdom in 1998, 13\% were malnourished based on BMI and weight loss and only these patients were referred to the Dietetic Department. In a recent British study of 2,283 patients, malnutrition was determined by weight loss, poor intake, wounds and infections. Prevalence decreased from $23.5 \%$ in 1998 to $19.1 \%$ in 2003 and referrals to the dietetic department increased after staff education and improvement of food quality (Peakeet al, 2005).

\subsection{Enteral Nutrition}

Enteral nutrition can be provided either orally or by tube feeding. By definition, Enteral means "within" or by the way of the gastrointestinal tract. It is the preferred route for nutritional support since the gut barrier and immune functions are preserved and systemic infections and other complications are reduced (Carr et al, 1996). Many studies support the implementation of enteral nutrition as soon as possible after resuscitation. Enteral feeding requires adequate gastric motility and a gastric residual volume in excess of $150 \mathrm{ml}$ will usually require feeding solutions to be administered slowly. However, it is not necessary to have bowel sounds for successful enteral nutrition. (Macfie, 2000).

Enteral feeding is administered to patients in the following conditions:

1. Those who cannot swallow due to paralysis of the muscles of swallowing (diphtheria, poliomyelitis) or cancer of the oral cavity or larynx.

2. Those who cannot be persuaded to eat

3. Those with persistent anorexia requiring forced feeding

4. Semiconscious or unconscious patients

5. Severe mal-absorption requiring administration of unpalatable formula

6. Short bowel syndrome

7. Those who are undernourished or at risk of becoming so

8. Those who cannot digest and absorb

9. Post-surgery

10. Patients with neurological and renal disorders and those with fevers or diabetes

11. Severe diarrhoea

\subsubsection{Oral Supplements}

Commercially available supplementary sip feeds are used in patients who can drink but in whom appetite is impaired. These feeds typically provide $200 \mathrm{kcal}$ and $2 \mathrm{~g}$ of nitrogen in each $200 \mathrm{ml}$ carton (Srilakshmi, 2011).

In order to reduce the risk of aspiration, tubes should not be placed in the stomach unless there is normal gastric emptying and an intact gag reflex and if possible, nasoenteric rather than nasogastric feeding tubes should be employed. This may be valuable in post-operative and critically ill patients in whom gastric motility is impaired.

A satisfactory tube feeding must be

1. Nutritionally adequate

2. Well tolerated by patient so that vomiting is not induced

3. Easily digested with no unfavourable reactions such as distension, diarrhoea or constipation

4. Easily prepared, and

5. Inexpensive

\subsubsection{Types of Enteral Foods Natural Liquid Foods}

Foods like whole or skim milk, eggs and some form of carbohydrate such as cooked strained cereals, sugar or molasses can be given. Vegetable oil or cream and non-fat dry milk are also incorporated to increase the caloric and protein levels respectively, foods like "complan" can be added to different foods to increase the nutritive value. 
Incidence of iatrogenic malnutrition in patients on enteral nutrition in the University of....

\section{Blenderized Foods}

Here, the ordinary food items which cannot be swallowed are blended to make thin liquid which can pass through nasogastric tube.Blenderized diets are well tolerated and are only infrequently associated with diarrhoea. They are less expensive than commercial formulas.

In Blenderized foods,

1. Water is added to make the volume to $1500 \mathrm{ml}$

2. Gives 1500 calories

3. Each ml provides one calorie

4. Gives $50 \mathrm{~g}$ protein

5. Cost is no more than an average meal in hospital

Table 1: Composition ofblenderized food for tube feeding for hospital practice

\begin{tabular}{llll}
\hline Ingredient & Amount(g) & Calories(kcal) & Protein(g) \\
\hline Rice & 100 & 345 & 6.8 \\
Green gramdal & 40 & 140 & 8.8 \\
Bread & 20 & 50 & 1.6 \\
Milk & $200 \mathrm{mls}$ & 234 & 8.6 \\
Skim milk powder & 60 & 215 & 23.0 \\
Spinach & 50 & 13 & 1.0 \\
Pumpkin & 50 & 12 & 0.7 \\
Carrots & 50 & 24 & 0.4 \\
Banana & 70 & 81 & 0.8 \\
Sugar & 60 & 240 & - \\
Refined oil & 20 & 180 & - \\
Butter & 7 & 51 & - \\
\hline
\end{tabular}

Source: Kawli, Sreenivasan, Eapen and Pradhan (1982),pp. 226-230

\section{Elemental Diets}

Normal foods may be replaced by preparation of protein or amino acids, fats and carbohydrates in easily assimilable forms. Such preparations are known as elemental diets. For tube feeding, commercially supplied polymeric mixtures can also be used. "Complan" is a commercial mixture of milk protein casein, carbohydrates (lactose) and fat. "Complan" can be given to supply 2000kcal of energy (Srilakshmi, 2011).

The commercial preparations possess the advantage of convenience, constant composition, presterilization, minimal preparation time and ease of administration. The disadvantages are high cost and unpleasant taste and sometime high osmolarity. Patients with pancreatic insufficiency, malabsorption or massive bowel resection may require an elemental formula.

\subsubsection{Feeding Requirements}

A concentration of about $1 \mathrm{kcal}$ per millilitre is satisfactory. Lesser concentration increases the volume which must be given to meet the nutrient and energy needs. A concentration of $1.5 \mathrm{kcal} / \mathrm{ml}$ is given for patients with increased caloric needs or patients with fluid restrictions. Greater concentration is more likely to produce diarrhoea and may be too thick to pass through the nasogastric tube.

Table 2: Feeding requirements in tube feeding

\begin{tabular}{ll}
\hline Nutrient & Amount \\
\hline Fluids & $30 \mathrm{ml} / \mathrm{kg}$ \\
Energy & $32 \mathrm{kcal} / \mathrm{kg}$ \\
Protein & $1 \mathrm{~g} / \mathrm{kg}$ (body weight if there are no external losses) \\
Sodium & $30-40 \mathrm{mmol}$ \\
Potassium & $1 \mathrm{mmol} / \mathrm{g}$ of protein \\
\hline
\end{tabular}

Source: Passmore and Eastwood (1986)

There are different infusion techniques and they include:

\section{Continuous infusion method}

This method is the administration of a fluid into a blood vessel usually over a prolonged period of time. It usually consists of small pulses of infusion, usually between 500 nanoliters and 10 millilitres, depending on the pump's design, with the rate of these pulses depending on the programmed infusion speed.

\section{Intermittent infusion method}

It is used when a patient requires medications only at certain times, and does not require additional fluid. It can use the same technique as an intravenous drip, but after the complete dose of medication has been given, the tubing is disconnected from the IV access device and the medication is injected directly (slowly, if it might irritate the vein or cause a too-rapid effect)it has a high infusion rate, alternating with a low 
programmable infusion rate to keep the cannula open. The timings are programmable. This mode is often used to administer antibiotics or other drugs that can irritate a blood vessel.

\subsection{Nutritional Assessment}

Nutrition assessment and screening of patients prior and on admission should occur within the first 24hours of admission. Screening criteria usually include weight and height, recent weight change, oral intake, excessive alcohol intake, and sometimes diagnosis (Mueller et al, 2007). A review of past medical history, physical examination, biochemical assessment and a detailed diet history provides insight into a patient's baseline nutritional status.

Whitney and Randy (2008) defined Nutritional Assessment as a comprehensive analysis of a person's nutritional status that uses health, socioeconomic, drug and histories, anthropometric measurements, physical examination and laboratory or biochemical tests. Malnutrition signs are easy to miss, they resemble the symptoms of other diseases e.g., diarrhoea, skin rash, pain and the likes. A person who has learned how to use assessment techniques to detect malnutrition can tell when these conditions are caused by poor nutrition and can take steps to correct them (Whitney \&Randy, 2008). Therefore, to detect malnutrition in individuals, health care professional use four nutritional assessment methods:

\subsubsection{Historical Information}

This is a way of evaluating nutrition status. It is used to obtain information about a person's history with respect to health status, socioeconomic status, drug use and diet. The health history reflects the person's medical records and may reveal a disease that interferes with the person's ability to eat or the body to use nutrients. The patient's family history or major disease is also needed, especially for conditions such as heart disease. Economic circumstances, social factors such as marital status, ethnic background and educational level also influence food choices and nutritional status.

\section{A sample nutritional history format}

1. How many meals and snacks do you eat each day?

Meals --------- Snacks

2. How many times a week do you eat the following meals away from home?

$$
\text { Breakfast --------- Lunch -------- Supper }
$$

3. What types of eating places do you frequently visit? (Check all that apply)

Fast-food --------- Supper/cafeteria

Restaurant ---------- Other

4. On average, how many pieces of fruit or glasses of juice do you eat or drink each day?

Fresh fruit --------- Juice (8 oz cup) ---------

5. On average, how many servings of vegetables do you eat each day?

6. On average, how many times a week do you eat a high- fibre breakfast cereal? ---------

7. How many times a week do you eat red meat (beef, lamb, veal) or pork?

8. How many times a week do you eat chicken or turkey?

9. How many times a week do you eat fish or shellfish?

10. How many hours of television do you watch every day?

11. Do you usually snack while watching television? Yes -

12. How many times a week do you eat desserts and sweets?

13. What types of beverages do you usually drink? How many servings of each do you drink a day?

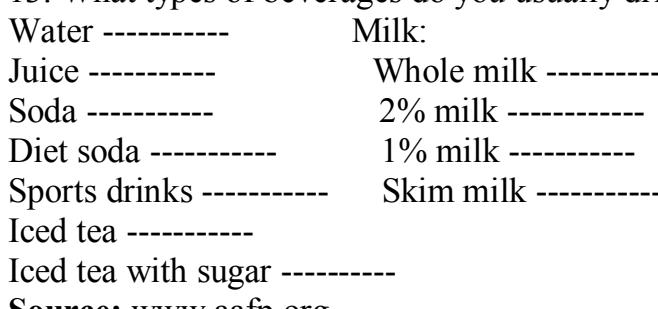

Alcohol:

Beer -

Wine

Hard liquor

Source: www.aafp.org.

\subsubsection{Anthropometric Data}

This helps to reveal nutrition problems. It involves measurement of physical characteristics of the body, such as height, weight, tricep, skin-fold thickness, head and arm circumferences, waist and hip ratios. The assessor compares measurement taken on an individual with standards specific for gender and age or with previous measures on the same individual. Measurement out of line with expectations may reveal such problems 
as growth failure in children, wasting or swelling of body tissue in adults and obesity conditions that may reflect energy or nutrient deficiencies or excesses.

\section{Anthropometric Classification of Nutrition}

There are five nutritional classifications:

1. Normal

2. Low height-for-age (stunted)

3. Low weight-for-height (wasted)

4. Low height-for-age and low weight-for-height (stunted and wasted)

5. Overweight

\subsubsection{Physical Examinations}

This nutritional assessment technique looks for clues to poor nutritional status. This is used to detect physical signs associated with nutrient deficiency, toxicity, and imbalance or even non- nutrition conditions. Sites like hair, eyes, skin, tongue and fingernails are thoroughly examined to check for malnutrition signs and symptoms. The posture of a patient may also be considered. This technique may also be used to confirm data collected from other assessment measures.

\begin{tabular}{ll}
\multicolumn{1}{c}{ Nutritional signs of Malnutrition and Good Health } \\
\hline Malnutrition & Good Health \\
\hline Poor weight gain, weight loss & Healthy skin and shiny hair \\
Weakness of muscles and fatigue & Healthy weight \\
Tiredness and lack of energy & Normal blood pressure level \\
Increased susceptibility to infections & Healthy cholesterol \\
Delayed and prolonged healing of even small wounds and cut & Clear vision \\
Irritability and dizziness & Mental alertness \\
Dry skin and hair & Regular bowel movement \\
Persistent diarrhoea or long term constipation & Good muscle tone \\
Irregular menstruation or depression & Healthy bones \\
\hline
\end{tabular}

Source:www.fitday.com/fitness-articles/nutrition/healthy-eating/good-nutrition.html

\subsubsection{Laboratory/ Biochemical Analysis}

The biochemical analysis uses samples like blood or urine which contain nutrients, enzymes, and metabolites that reflect nutritional status. It is also used to check electrolyte imbalance and nutrient deficiencies. The results obtained are compared with normal values for a similar population. A goal of nutrition assessment is to uncover early signs of malnutrition before symptoms appear, and laboratory tests are most useful for this purpose. They can also confirm suspicions raised by other assessment methods.

Table of Biochemical test of good nutrition

\begin{tabular}{ll}
\hline Nutrients & Values \\
\hline Glucose & $117 \mathrm{mcg} / \mathrm{dl}(80-120)$ \\
BUN(blood urea nitrogen) & $24 \mathrm{mg} / \mathrm{dl}(8-29)$ \\
Creatinine & $0.8 \mathrm{mg} / \mathrm{dl}(0.4-1.2)$ \\
Sodium(Na) & $140 \mathrm{mEq} / 1(10-118)$ \\
Potassium(K) & $5.2 \mathrm{mEq} / 1(4.4-6.1)$ \\
Chloride & $104 \mathrm{mEq} / 1(10-118)$ \\
CO$_{2}($ carbon dioxide) & $22 \mathrm{mEq} / 1(22-285)$ \\
Calcium & $9.6 \mathrm{mg} / \mathrm{dl}(9.4-11.6)$ \\
Phosphorus & $5.6 \mathrm{mg} / \mathrm{dl}(2.5-6.2)$ \\
Total Protein(TP) & $6.3 \mathrm{gm} / \mathrm{dl}(5.8-8.1)$ \\
Albumin & $2.9 \mathrm{gm} / \mathrm{dl}(2.6-4)$ \\
Bilirubin & $0.6 \mathrm{mg} / \mathrm{dl}(0.2-0.7)$ \\
Cholesterol & $204 \mathrm{mg} / \mathrm{dl}(129-330)$ \\
Triglyceride & $82 \mathrm{mg} / \mathrm{dl}(36-135)$ \\
ALKP(alkaline phosphatase) & $65 \mathrm{U} / 1(20-70)$ \\
AST(aspirate aminotransferase) & $30 \mathrm{U} / 1(14-42)$ \\
ALT(alanine aminotransferase) & $45 \mathrm{U} / 1(15-52)$ \\
GGT(gamma-glutamyltransferase) & $5 \mathrm{U} / 1(1-12)$ \\
Amylase & $850 \mathrm{U} / 1(280-950)$ \\
CK (creatine kinase) & $47 \mathrm{U} / 1(0-130)$ \\
\hline
\end{tabular}

Source: www.9sites.org

Each of these methods involves collecting data in various ways and interpreting each finding in relation to others creates a total picture (Whitney \&Randy, 2008). The National Nutrition Monitoring and Related Research Act on Nutrition mandated that hospitals establish nutrition screening and assessment guidelines. It 
recommended that nutrition screening should occur within the first 24 hours of admission. Screening criteria usually include weight, height, recent weight change, oral intake and sometimes diagnosis (Krystofiaket al, 2007).

\section{Nutrition Screening and Assessment Guideline}

Screening has to address three or four basic questions:

1. Recent weight loss - is $\mathrm{BMI}<20.5$ ?

2. Current body mass index - has the patient lost weight since on admission?

3. Disease severity - is the patient severely ill? (e.g. in intensive therapy)

4. Recent food intake - has the patient had a reduced dietary intake in the last week?

\subsection{Causes of Iatrogenic Malnutrition}

Many patients are already malnourished at the point of admission while others become malnourished during their hospital stay. The aetiologies include alterations in the intake, digestion, absorption and/or metabolism of food. Risk factors include gastrointestinal disorders, chronic diseases, malignancies, low socioeconomic status, psychological disorder, old age, alcohol and drug abuse (Correiaet al, 2003). Several disease states and acute events can predispose patients to malnutrition, the degree of which is usually determined by the severity of the illness. (ASPEN, 2002). The most obvious are those that prevent oral food intake such as cancer, tumours or strictures in the oesophagus, stroke and degenerative neurologic disorders that result in dysphagia. Conditions such as chronic obstructive pulmonary disease, chronic infections and cancer can result in increased metabolic demand and weight loss due to cachexia and poor oral intake. Illnesses always decrease patient appetite as a result of pains nausea, weakness and altered mood or mental status. They can be dissatisfied with repetitive menu cycle, dietary restriction, and the food which can be the type they prefer (Clare et al, 2002).

The following are the causes of iatrogenic malnutrition in hospitalized patients.

\subsubsection{Activities of Healthcare Professionals}

Malnutrition increases in patients during hospitalization. According to the "Low Rate of NutritionRelated Disorders", one potential cause of worsening nutritional status may be inadequate medical staff awareness about the importance of nutrition in hospitalized patients(Saeed et al, 2006). Deborah(2005) revealed that lack of attention to functional impairment by physicians to patients upon admission and adverse drug effects are the most common causes of iatrogenesis. Adverse drug effects account for approximately $15 \%$ of hospital admission in patients over 60 years old as compared to $60 \%$ for younger patients. About $62 \%$ of adverse drug effects resulting in hospital admission are potentially preventable and $25 \%$ may be life threatening. Majority may be due to adequate drug therapy, monitoring therapy or inadequate dosage.

Clinicians sometimes hold enteral nutrition out of suspicion that it causes diarrhoea, when infant diarrhoea is due to other factors such as the underlying illness, altered anatomy, infection and medication.

Green (1999) stated that health care professionals contribute to disease- related malnutrition in the following ways:

1. Failure to recognize and document the problem.

2. Obsolete practices relating to nutritional screening assessment and support.

3. Unclear accountability, leadership, inadequate assistance and support at most times in hospital.

All these are as a result of lack of knowledge and practical training, lack of evidence-based standards and guidelines, lack of specialists in clinical nutrition and lack of awareness of services available.

\subsubsection{Length of Hospital Stay}

The nutritional status of patients is known to worsen during hospital stay which is partly due to poor recognition by the medical staff and adverse clinical routine (Kritinaet al, 2008). Length of hospital stay is significantly longer in malnourished patients and higher treatment costs are reported in malnutrition. Patients who are not motile (non-ambulatory) over a period of time experience muscle wasting due to weakness of the muscle and cell depletions.

\subsubsection{Inadequate Nutrient Intake}

Nutritional intake of patients often decreases during hospitalization.

Patients are required to be on Nil Per Oral (NPO) prior to many investigations and procedures, as well as before and after surgery. Delay of the need for several procedures result in prolonged periods without nutrition. Patients' appetite usually decrease during illness due to pain, nausea, weakness and altered mood or mental status, and they can become dissatisfied with repetitive menu cycles, dietary restriction, and the food which may not be the type they prefer (Kondrup, et al, 2002). Most commonly, malnutrition is caused by a 
combination of decreased oral intake due to anorexia and increased nutrient requirement due to underlying disease. (Escott- Stump, 2008). Diseases that prevent oral food intake include oral cancer, tumors or strictures in the throat or oesophagus, stroke and degenerative neurologic disorders that result in dysphagia, trauma and others who are ventilator dependent rely on the timely initiation of nutrition support.

Nutrient losses may occur through the skin in certain dermatological diseases characterized by desquamation and exudation of fluids rich in electrolyte and protein as well as in severe burns cases. Intestinal fistulas are inflammatory diseases of the digestive tract or maybe as a result of surgery, resulting in high losses of nutrients, water and electrolytes.

Patients with gastrointestinal disorders are among those who are most prone to developing malnutrition. Patients with gastroenteritis, gastric outlet or bowel obstruction and disorders present with varied degrees of malnutrition depending on how long they waited to seek medical care. Surgical resections of the gastrointestinal (GI) tract for cancer or Cohn's disease can result in severe mal-digestion and malabsorption of nutrients and can cause digestive disorders such as cystic fibrosis. Gastric by-pass procedures while effective for weight loss predispose patients to serious micronutrient deficiencies. Chronic liver disease can contribute to poor nutrient digestion and absorption, patients with pancreatitis often present with malnutrition (Green, 1999). In a population of patients preparing for GI or hernia surgery, the use of Subjective Global Assessment identifies malnutrition in half of the patients with almost $20 \%$ being severely malnourished (Correia, 2001).

Hyper- metabolic states are characterized by an acceleration of metabolic processes caused by the disease. Major surgery involves greater intensity and duration that will increase catabolic effects. Disorders associated with very severe or prolonged disease conditions, lead to a hyper-metabolic stress response from the body, thus altering metabolism and fuel utilization. The extent of alteration depends on severity and the type of stress involved. Fever increases the basal metabolism by a little more than $10 \%$ for each degree temperature rise. Elective operations increase resting energy expenditure by up to $10 \%$, multiple fractures, stab wounds, or gunshot wounds cause an increase up to $30 \%$, severe sepsis up to $60 \%$. Extensive third-degree burn can even cause more than a doubling of the energy expenditure. Conditions such as chronic obstructive pulmonary disease, chronic infections and cancer can result in increased metabolic demand and weight loss due to cachexia and poor oral intake (American Society of Parenteral and Enteral Nutrition, ASPEN, 2007).

\subsection{Effects/Complications of Malnutrition}

Malnutrition is characterized by an impairment of a number of physiological functions. Respiratory muscle functions are deteriorated as are other bodily functions such as cardiac, sexual and immune functions as well as thermoregulation. Malnutrition leads to depression, irritability, anxiety, reduced ability to maintain concentration and reduced sexual drive. In children, malnutrition impairs growth and delays sexual maturation (Morley \& Lucas, 1993). "Protein- Energy Malnutrition has been associated with a number of health consequences including decreased bone mass, immune dysfunction, anaemia, reduced cognitive function and poor wound healing" (Doniniet al, 2003).

Under nutrition in critical periods impairs the development of normal immune system leading to more chronic and frequent infections (Genninggham-Rundles et al, 2005). Lack of adequate macronutrients or some micronutrients (like zinc, selenium, iron and the antioxidant vitamins) can lead to clinical significant immune deficiency and infections in children (Ibid). A woman with low body mass index (BMI $<18 \mathrm{Kg} / \mathrm{m}$ ) may have difficulty becoming pregnant and preconception under nutrition shortens gestation in women who do become pregnant. (Ryacoet al, 2005).

It is known that adequate nutrition is needed for many important aspects of brain functioning and it has been shown that optimal nutrition promotes both functional health status and mental wellbeing (Drewnowskiet $a l, 2001)$. Malnutrition is however, a complex problem and is not limited to protein deficiency. Micronutrient malnutrition such as deficiency of a particular vitamin or mineral can have a range of adverse health effects. It is important to consider the psychological effects such as depression, anxiety, irritability, apathy, sleep pattern and loss of concentration (Stanza et al, 2007)

\subsubsection{Effects of Iatrogenic Malnutrition}

According to the British Dietetics Association (2006), malnutrition in hospital causes a reduction in muscle and body tissue mass and also results in altered metabolic and physiological function which has adverse effects on health. Muscle wasting and weakness decrease mobility and stamina and impair functions of the lungs and heart. Malnutrition is also associated with poor wound healing, impaired immune response and delayed recovery from illness with a higher incidence of post-operative complications. Correcting under nutrition has many health benefits including improved recovery from diseases and illness with fewer complications and short hospital stay. Malnutrition can lead to weight loss, poor wound healing, decreased intestinal motility, anaemia, oedema or dehydration and the presence of ulcers. The circulatory blood volume and the concentration of the serum proteins, haemoglobin and electrolytes may be reduced (Srilakshmi, 2011). 


\subsubsection{Complications of Enteral Nutrition}

Enteral which is generally known as tube feeding, may produce several complications in a patient if used for a long time. These complications include: aspiration into the lungs of stomach contents, oesophageal erosion, vomiting, bloating, cramps, fluids and electrolytes imbalance, hyperglycaemia, congestive heart failure, hyperosmolar coma, and essential fatty acid deficiency. A weight gain of over $0.7 \mathrm{~kg}$ per day indicates excess of extracellular fluid which may lead to congestive heart failure. Serum electrolytes, glucose and urea must be periodically checked. Cough reflex may be depressed as in old or infirm patient or in-patients with pulmonary problem. Diarrhoea may be secondary to rapid feeds, hyperosmolar feed solutions, and lactose deficiency in a patient fed with milk or milk products or associated antibiotic therapy (Antia\& Abraham, 2002).

Other complications of tube feeding include:

1. Injury or ulceration of nasal, buccal or pharyngeal mucosa.

2. Perforation of hypo pharynx, oesophagus, trachea, bronchus, lung, pleural cavity, or mediastinum.

3. Coiling of tube in mouth, oesophagus, hiatal hernia, or stomach.

4. Blockage of tube with clotted formula, undissolved medications, or foreign bodies.

5. Rupture of tube during flushing or attempt to clear blockage.

6. Loss of tube into gastrointestinal tract.

7. Reverse or downward migration of tube, gastric outlet obstruction.

8. Accidental or intentional extubation necessitating replacement.

9. Enlargement of enterostomy stoma with leakage and skin irritation.

10. Aspiration

11. Infections of sinuses and middle ear

12. Compromise of lower oesophageal sphincter, and reflux oesophagitis

13. Upper gastrointestinal bleeding.

14. Emotional distress due to changed body image.

15. Loss of patient autonomy and mobility with use of restraints.

16. Disturbances of fluid and electrolyte balance.

17. Inadequate nutrient administration.

18. fistulas

19. intraperitoneal leakage

20. Formation of granulation tissue around stoma. (Mobarhan and Trumbore, 1991).

Another complication of enteral nutrition is "refeeding syndrome". This is simply refeeding of severely malnourished patient which may result in acute intracellular shift of electrolytes as cell anabolism is stimulated. Refeeding can cause acute decrease in the circulatory levels of potassium, magnesium and phosphorus. Hence these electrolytes should be monitored and replaced as needed to maintain normal circulating levels by supplementation. (Srilakshmi, 2011).

\subsection{Treatment/Management of Iatrogenic Malnutrition in patients on Enteral Nutrition}

Early detection of malnutrition by routine screening of vulnerable groups (those with chronic diseases and the elderly) can do much to identify those who would benefit from dietary support measures and nutrition intervention. Treatment of malnutrition usually consists of replacing missing nutrient, treating symptoms needed and treating any underlying medical conditions. Managing nutritional status starts with a nutritional assessment. This enables a clinical nutrition or registered dietician to confirm the presence of malnutrition, assess the effect of the disorder and formulate diets that will restore adequate nutrition (Kraft et al, 2005).

In case of patients who can eat orally, between meal snacks and liquid nutrients supplements can be helpful. The development of menus providing nutritionally adequate and appealing meals that the patient can eat and where necessary, supply additional food and nutrient supplement may be all that is needed for some patients while others may require more intensive artificial nutritional support through a tube or intravenously. (British Dietetics Association, 2006). Children with severe acute malnutrition need to be treated with specialized therapeutic diets - F75, F100 or HEMIX, alongside the diagnosis and management of complications during inpatient care (WHO, 2002). Treatment emphasizes frequent nutrient dense energy meals like, HEMIX and resolution of underlying causes of malnutrition, poverty, infection and illness.

Patients who cannot or will not at or who are unable to absorb nutrients taken by mouth may be fed intravenously (ASPEN, 2002). According to the American Society for Parenteral and Enteral Nutrition ASPEN guidelines (2002), patients who are either unable or expected to be unable to take sufficient oral nutrition for 7 to 14 days are candidates for nutrition support. Enteral nutrition should be used if the gastrointestinal tract is functional and Parenteral nutrition should be used only if the gastrointestinal tract is not functioning or cannot be accessed (Grover et al, 2008). 
Studies have shown that enteral nutrition has the following advantages:

1. Maintenance of the structural and functional integrity and weight of the intestines

2. Aid in better insulin response to food absorbed through the intestine

3. Does not easily produce electrolyte and trace element imbalances

4. Improves resistance to infections in burns, burn-induced hyper-metabolic response

5. Maintains intestinal structure and function

6. Is effective during liver transplantation and the elemental diet helps to tide over in short-bowel syndrome until functional regeneration occurs in the residual bowel.

7. It is also simple, inexpensive, avoids catheter sepsis and infections,

8. Convenient and easily tolerated (Antia\&Abraham, 2002)

Refeeding risk is the most critical aspect early in the treatment of malnourished patients especially those on enteral and Parenteral nutrition. It is important that clinicians initiate feeding at low calorie levels while monitoring serum electrolytes. An intracellular shift of potassium, magnesium and phosphorus can result in dangerously low serum levels, thus clinicians should monitor at least daily with the onset of feeding and replete electrolytes when necessary. Avoidance of excessive fluid administration is also important especially in patients with cachexia. Provision of multivitamin or single nutrient supplements or at least Thiamine repletion is often necessary (Kraft et al, 2005). Refeeding syndrome complications can be avoided by anticipating the problem and initially feeding only $20-30 \mathrm{kcal} / \mathrm{kg}$, checking initial serum levels of potassium, magnesium and phosphate (Srilakshmi, 2011).

A study describing all hospitalized patients reported that $70 \%$ of the patients did not reach a reasonable recommended daily nutrient intake. In most cases, simple measures such as an increased protein and energy intake, is known to have a significant beneficial effect in decelerating the development of malnutrition. An early Swiss study showed that a meagre daily supplement of $20 \mathrm{~g}$ protein and 254 calories reduced morbidity among ill patients, whether in hospital or not, with attendant significant decrease in length of hospital stay, complications and mortality rates. Similar results have been shown in surgical patients, thus demonstrating that malnutrition in hospital patients can be reduced by appropriate and early identification of the at-risk patient, and that adequate feeding as part of the patient's overall therapy is a cost-effective practice. Patients who require elective intestinal surgery should receive nutritional assessment before their operation (Srilakshmi, 2011).

Despite the realization of hospital malnutrition more than 30 years ago, it remains a significant problem. Many ailments or injuries for which patients are admitted to the hospital predispose them to malnutrition as does hospitalization itself (Kelly et al, 2005). The final solution to malnutrition in hospitals probably lies in recognizing human nutrition as a discrete discipline in which all medical graduates should reach minimum level of competence, and some will specialize. Compared with other health care practitioners, Registered Dietitians (RDs) are the most qualified to recognize malnutrition as well as to help prevent and treat it. Prompt identification, treatment and monitoring of malnourished patients as well as helping to educate other health care professionals, will continue to make a positive impact on healthcare delivery (WHO, 2002).

\subsection{Study Area}

\section{Methodology}

University of Nigeria Teaching Hospital Ituku-Ozalla, Enugu was used as the site for this project work. This hospital is located in the Ituku/Ozalla community along the Enugu-Okigwe express way. The hospital was established in the early $20^{\text {th }}$ century as a standard general hospital for Africans, built by the colonial masters. It was however, changed to a general hospital then under the Government of the East Central State. It was transferred to a specialist hospital on July 1, 1970. Today the hospital is in its permanent site and covers an area of about 200 acres of land. It has a bed capacity of over 500 beds and tremendous total number of professional and non-professional personnel. This hospital is characterized by its serene and conducive environment which makes it possible for patients and workers to thrive and go about their respective business.

\subsection{Sample Population}

The subjects in this study were inpatients on enteral feeds in the University of Nigeria Teaching Hospital Ituku-Ozalla, Enugu.

\subsection{Data Collection Method}

Structured and validated questionnaires were administeredto patients on enteral feeding in the hospital. The questionnaire contained patient's personal information, Anthropometric data, Dietary Assessment, Nutrition-related problems/Diagnosis. 
Incidence of iatrogenic malnutrition in patients on enteral nutrition in the University of....

\subsection{Anthropometry}

The anthropometric measurements involving heights and weights were carried out on the respondents and BMI calculated

For ambulatory patients:

$\mathrm{BMI}=\frac{\text { Weig ht in kilograms }(\mathrm{kg})}{\text { Heig ht in meters square }(\mathrm{m} 2)}=\mathrm{kg} / \mathrm{m}^{2}$

With the BMI calculated, it was compared to WHO (1997) classification.

For non-ambulatory patients, the body weight was estimated using knee height and mid-arm circumference for various groups thus:

Females

6-18yrs $\mathrm{Wt}=(\mathrm{KHx} 0.71)+(\mathrm{MACx} 2.59)-50.43$. Accuracy $= \pm 7.65 \mathrm{~kg}$

19-55yrs $\quad \mathrm{Wt}=(\mathrm{KHx} 1.24)+(\mathrm{MACx} 2.97)-82.48$. Accuracy $= \pm 11.98 \mathrm{~kg}$

60-80yrs $\quad \mathrm{Wt}=(\mathrm{KHx} 1.50)+(\mathrm{MACx} 2.58)-84.22$. Accuracy $= \pm 14.52 \mathrm{~kg}$

Males

6-18yrs $\mathrm{Wt}=(\mathrm{KHx} 0.59)+(\mathrm{MACx} 2.73)-48.32$. Accuracy $= \pm 7.50 \mathrm{~kg}$

19-55yrs $\quad \mathrm{Wt}=(\mathrm{KHx} 1.09)+(\mathrm{MACx} 3.14)-83.72$. Accuracy $= \pm 11.30 \mathrm{~kg}$

60-80yrs $\quad \mathrm{Wt}=(\mathrm{KHx} 0.44)+(\mathrm{MACx} 2.86)-39.21$. Accuracy $= \pm 7.04 \mathrm{~kg}$

Where: $\mathrm{KH}=$ knee height; $\mathrm{MAC}=$ mid arm circumference

Estimates of Height/recumbent length for the various groups

\begin{tabular}{lll}
\hline Age & Equation & Error \\
\hline Black females & & \\
$>60$ & $\mathrm{~S}=58.72+(1.96 \mathrm{KH})$ & $8.26 \mathrm{~cm}$ \\
$19-60$ & $\mathrm{~S}=68.19+(1.86 \mathrm{KH})$ & $7.60 \mathrm{~cm}$ \\
$6-18$ & $\mathrm{~S}=46.59+(2.02 \mathrm{KH})$ & $8.78 \mathrm{~cm}$ \\
Black males & & \\
$>60$ & $\mathrm{~S}=95.79+(1.37 \mathrm{KH})$ & $8.44 \mathrm{~cm}$ \\
$19-60$ & $\mathrm{~S}=73.42+(1.79 \mathrm{KH})$ & $7.20 \mathrm{~cm}$ \\
$6-18$ & $\mathrm{~S}=39.60+(2.18 \mathrm{KH})$ & $9.16 \mathrm{~cm}$ \\
\hline
\end{tabular}

Source: Nutritional Assessment

\subsection{Method of Data Analysis}

The data was analysed using simple frequencies, simple percentages and frequency tables.

\section{Results}

The results of the study conducted on a sample of twenty patients on enteral nutrition in the University of Nigeria Teaching Hospital, Enugu, Nigeria, over a period of one month are noted in tables as follows:

Table I: Patients' Information

\begin{tabular}{|l|l|l|l|l|}
\hline & Frequency & Percentage & $\begin{array}{l}\text { Cumulative } \\
\text { frequency }\end{array}$ & $\begin{array}{l}\text { Cumulative } \\
\text { percentage }\end{array}$ \\
\hline $\begin{array}{l}\text { Age (yrs) } \\
0-10\end{array}$ & 3 & & 3 & 15 \\
\hline $11-20$ & 1 & 15 & 4 & 20 \\
\hline $21-30$ & 2 & 5 & 6 & 30 \\
\hline $31-40$ & - & 10 & 6 & 30 \\
\hline $41-50$ & 3 & - & 9 & 45 \\
\hline Above 50 & 11 & 15 & 20 & 100 \\
\hline Total & $\mathbf{2 0}$ & 55 & & \\
\hline Sex & & $\mathbf{1 0 0}$ & 12 & 10 \\
\hline Male & 12 & & 20 & \\
\hline Female & 8 & 60 & & \\
\hline Total & $\mathbf{2 0}$ & 40 & & \\
\hline Date ofadmission(Month) & & $\mathbf{1 0 0}$ & & 5 \\
\hline April & 1 & & 1 & 20 \\
\hline May & 3 & 5 & 4 & 50 \\
\hline June & 6 & 15 & 10 & 100 \\
\hline July & 10 & 30 & 20 & \\
\hline Total & $\mathbf{2 0}$ & 50 & & \\
\hline
\end{tabular}

Table 1 showed that $55 \%$ of the patients were above 50 years of age while $15 \%$ were between the ages of $0-10$ years. $60 \%$ of the patients were male while $40 \%$ were females. About $50 \%$ of the patients were admitted in July and $30 \%$ in June. 
Table II: Anthropometric Measurement

\begin{tabular}{|c|c|c|}
\hline & f & $\%$ \\
\hline \multicolumn{3}{|l|}{ Height (m) } \\
\hline $0.01-0.50$ & 1 & 5 \\
\hline $0.51-1.00$ & 2 & 10 \\
\hline $1.01-1.50$ & 2 & 10 \\
\hline $1.51-2.00$ & 15 & 75 \\
\hline Total & 20 & 100 \\
\hline \multicolumn{3}{|c|}{ Current Weight(kg) } \\
\hline $1-10$ & 3 & 15 \\
\hline $11-20$ & - & - \\
\hline $21-30$ & - & - \\
\hline $31-40$ & 3 & 15 \\
\hline $41-50$ & 10 & 50 \\
\hline $51-60$ & 3 & 15 \\
\hline Above 60 & 1 & 5 \\
\hline Total & 20 & 100 \\
\hline \multicolumn{3}{|c|}{ Usual Weight (kg) } \\
\hline $1-10$ & 3 & 15 \\
\hline $11-20$ & - & - \\
\hline $21-30$ & - & - \\
\hline $31-40$ & - & - \\
\hline $41-50$ & 6 & 30 \\
\hline $51-60$ & 9 & 45 \\
\hline Above & 2 & 10 \\
\hline Total & 20 & 100 \\
\hline \multicolumn{3}{|c|}{ DesirableBody Weight(kg) } \\
\hline $1-10$ & 1 & 5 \\
\hline $11-20$ & 2 & 10 \\
\hline $21-30$ & - & - \\
\hline $31-40$ & - & - \\
\hline $41-50$ & 2 & 10 \\
\hline $51-60$ & 5 & 25 \\
\hline Above 60 & 10 & 50 \\
\hline Total & 20 & 100 \\
\hline \multicolumn{3}{|c|}{ Body Mass Index $\left(\mathrm{kg} / \mathrm{m}^{2}\right)$} \\
\hline$<18.5$ & 12 & 60 \\
\hline $18.5-24.9$ & 7 & 35 \\
\hline $25-29.9$ & 1 & 5 \\
\hline Total & 20 & 100 \\
\hline \multicolumn{3}{|c|}{ Percentage Desirable Body Weight (\%) } \\
\hline$<50$ & 1 & 5 \\
\hline $51-60$ & 2 & 10 \\
\hline $61-70$ & - & - \\
\hline $71-80$ & 11 & 55 \\
\hline $81-90$ & 5 & 25 \\
\hline $91-100$ & - & - \\
\hline Above 100 & 1 & 5 \\
\hline \multicolumn{3}{|c|}{ Percentage Body Weight Loss $(\%)$} \\
\hline $1-5$ & - & - \\
\hline $6-10$ & 10 & 50 \\
\hline $11-15$ & 7 & 35 \\
\hline $16-20$ & 2 & 10 \\
\hline $21-25$ & - & - \\
\hline Above 25 & 1 & 5 \\
\hline Total & 20 & 100 \\
\hline
\end{tabular}

Table II revealed that $75 \%$ of the patients' height fall between $1.51-2.00$ metres, $50 \%$ of the patients' have a current weight between $41-50 \mathrm{~kg}, 45 \%$ of the patients had a usual weight of $51-60 \mathrm{~kg}$ and $30 \%$ and $30 \%$ had a usual weight of $45-50 \mathrm{~kg}$. It was also showed that the desirable weight of $50 \%$ of the patients was above $60 \mathrm{~kg}$ while $25 \%$ had a desirable weight between $51-60 \mathrm{~kg}$. $60 \%$ of the patients had a body mass index less than 18.5(i.e. underweight) whereas only $35 \%$ had a body mass index between 18.5-24.9(normal), and only one patient (about 5\%) had a body mass index between 25-29.9(overweight). About 55\% of the patients had a percentage desirable body weight of $71-80 \%$ while $25 \%$ had a percentage desirable body weight of $81-90 \%$. Half of the patients $(50 \%)$ had a percentage weight loss of $6-10 \%$ while about $35 \%$ had a percentage weight loss of $11-15 \%$. 


\begin{tabular}{|c|c|c|}
\hline & f & $\%$ \\
\hline \multicolumn{3}{|c|}{ Haemoglobin $\left(\times 10^{9} / \mathbf{I}\right)$} \\
\hline Less than 5.0 & 3 & 15 \\
\hline $5.0-10.0$ & 15 & 75 \\
\hline Greater than 10.0 & 2 & 10 \\
\hline Total & 20 & 100 \\
\hline \multicolumn{3}{|c|}{ Fasting BloodSugar (mg/dl) } \\
\hline Less than 80 & - & - \\
\hline $80-120$ & 10 & 50 \\
\hline 120 above & 5 & 25 \\
\hline None & 5 & 25 \\
\hline Total & 20 & 100 \\
\hline \multicolumn{3}{|c|}{ Total Cholesterol(mmol/dl) } \\
\hline Less than 3.6 & 3 & 15 \\
\hline $3.6-6.2$ & 11 & 55 \\
\hline Greater than 6.2 & 1 & 5 \\
\hline None & 5 & 25 \\
\hline Total & 20 & 100 \\
\hline \multicolumn{3}{|c|}{ Low Density Lipoprotein (mmol/dl) } \\
\hline Less than 1.9 & 2 & 10 \\
\hline $1.9-3.6$ & 11 & 55 \\
\hline Greater than 3.6 & 2 & 10 \\
\hline None & 5 & 25 \\
\hline Total & 20 & 100 \\
\hline \multicolumn{3}{|c|}{ Very Low DensityLipoprotein (mmol/dl) } \\
\hline \multicolumn{3}{|l|}{ Less than 0.4} \\
\hline $0.4-1.3$ & 2 & 10 \\
\hline Greater than 1.3 & 10 & 50 \\
\hline None & 3 & 15 \\
\hline Total & 20 & 100 \\
\hline \multicolumn{3}{|c|}{ Triglyceride(mmol/dl) } \\
\hline Less than 0.5 & 4 & 20 \\
\hline $0.5-1.0$ & 7 & 35 \\
\hline Greater 1.0 & 4 & 20 \\
\hline None & 5 & 25 \\
\hline Total & 20 & 100 \\
\hline
\end{tabular}

In table III, $75 \%$ of the patients had haemoglobin values of $5.0-10.0 \times 10^{9}$, while about $15 \%$ had less than $5.0 \times 10^{9} / 1$. the fasting blood sugar of $50 \%$ of the patients was within $80-120 \mathrm{mg} / \mathrm{dl}, 25 \%$ of the patients had more than $120 \mathrm{mg} / \mathrm{dl}$ while none had less than $80 \mathrm{mg} / \mathrm{dl}$. The FBS of $25 \%$ of the patients were not recorded. $55 \%$ of the patients had total cholesterol value of 3.6-6.2 $\mathrm{mmol} / \mathrm{dl}$ while that of $25 \%$ of the patients was not recorded. The low density lipoprotein of $55 \%$ of the patients was within $1.9-3.6 \mathrm{mmol} / \mathrm{dl}$ while that of $25 \%$ of the patients was not recorded. $50 \%$ of the patients had a very low density lipoprotein of within $0.4-1.3$ whereas that of $25 \%$ of the patients was not recorded. The triglyceride level of $35 \%$ of the patients fall within $0.5-1.0 \mathrm{mmol} / \mathrm{dl}$, while $20 \%$ had a value greater than $1.0 \mathrm{mmol} / \mathrm{dl}$ and value of $25 \%$ of the patients were not recorded.

Table IV Clinical Assessment

\begin{tabular}{lll}
\hline & f & \% \\
\hline PALE & & \\
Present & 5 & 25 \\
Absent & 15 & 75 \\
Total & $\mathbf{2 0}$ & $\mathbf{1 0 0}$ \\
ANGULAR STOMATITIS & & \\
Present & 3 & 15 \\
Absent & 17 & 85 \\
Total & $\mathbf{2 0}$ & $\mathbf{1 0 0}$ \\
ODEMA & & \\
Present & 8 & 40 \\
Absent & 12 & 60 \\
Total & $\mathbf{2 0}$ & $\mathbf{1 0 0}$ \\
MUSCLE WASTING & & \\
Present & 7 & 35 \\
Absent & 13 & 65 \\
Total & $\mathbf{2 0}$ & $\mathbf{1 0 0}$ \\
\hline
\end{tabular}

In table IV, the clinical assessments showed that $25 \%$ of the Patients were pale while $75 \%$ were not, $15 \%$ had angular stomatitis while $85 \%$ had not, Oedema was present in $40 \%$ of the patients but not in the other $60 \%, 35 \%$ were wasted while $65 \%$ were not. 
Incidence of iatrogenic malnutrition in patients on enteral nutrition in the University of....

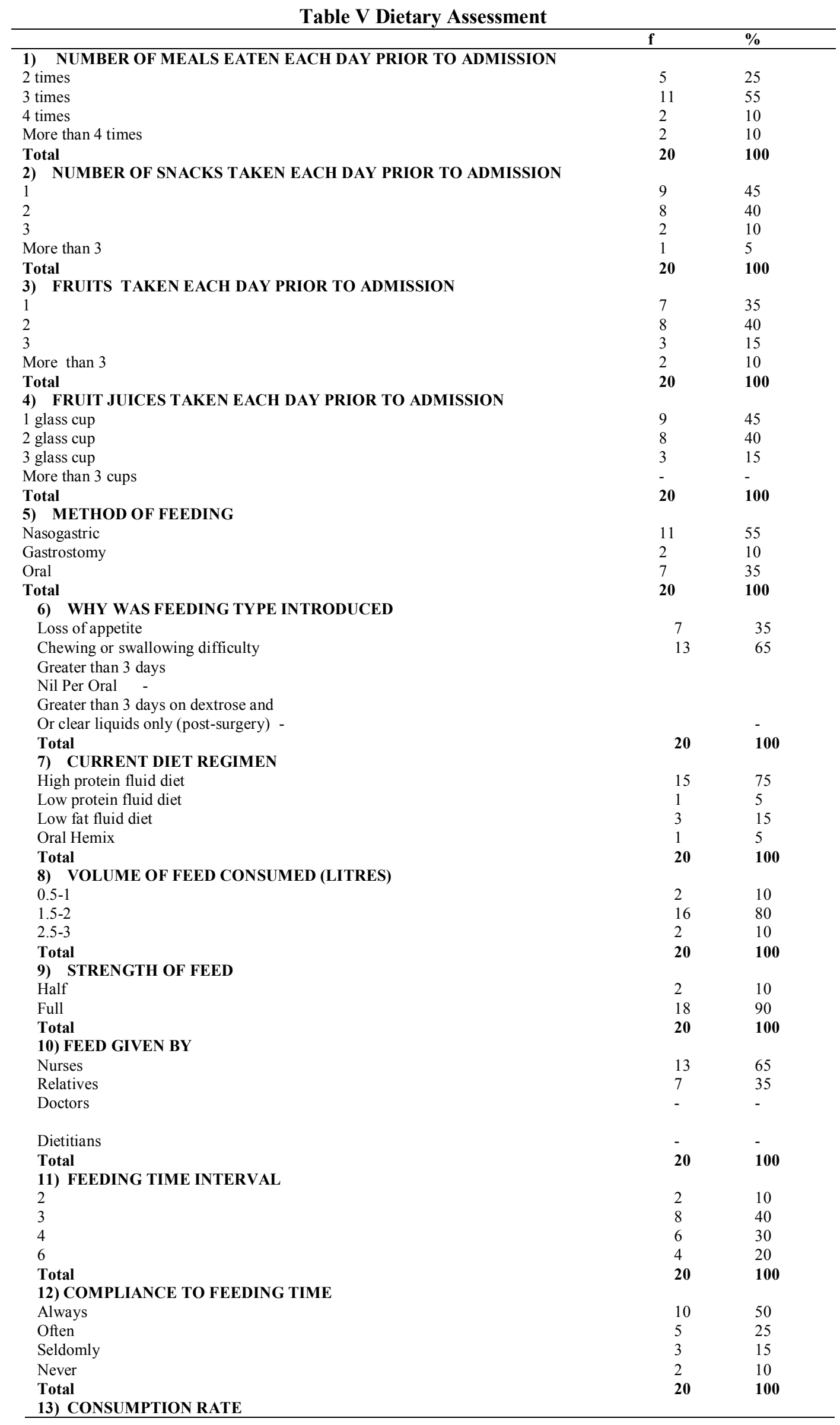


Incidence of iatrogenic malnutrition in patients on enteral nutrition in the University of....

\begin{tabular}{|c|c|c|}
\hline High & 8 & 40 \\
\hline Low & 10 & 50 \\
\hline Refuses & 2 & 10 \\
\hline Total & 20 & 100 \\
\hline \multicolumn{3}{|c|}{ 14) FEELINGS AFTER CONSUMPTION } \\
\hline Satisfied & 13 & 65 \\
\hline Hungry & 3 & 15 \\
\hline Calm & 4 & 20 \\
\hline Total & 20 & 100 \\
\hline \multicolumn{3}{|c|}{ 15) REACTIONS AFTER FEEDING } \\
\hline Vomiting & 1 & 5 \\
\hline Uneasy & - & 1 \\
\hline Diarrhea & 1 & 5 \\
\hline None & 18 & 90 \\
\hline Total & 20 & 100 \\
\hline \multicolumn{3}{|l|}{ 16) RECOMMENDED BY } \\
\hline Dietician & 20 & 100 \\
\hline Doctor & - & - \\
\hline Nurse & - & - \\
\hline Self & - & - \\
\hline Total & 20 & 100 \\
\hline \multicolumn{3}{|l|}{ 17) DATE COMMENCED } \\
\hline On admission & - & \\
\hline Few days after admission & 2 & 10 \\
\hline A week after admission & 7 & 35 \\
\hline Two weeks after admission & 8 & 40 \\
\hline More than two weeks & 3 & 15 \\
\hline Total & 20 & 100 \\
\hline
\end{tabular}

It can be seen in Table $\mathrm{V}$ that $55 \%$ of the patients ate meals 3 times daily and $25 \% 2$ times daily prior to admission $45 \%$ of the patients took 1 snack daily while $40 \%$ of the patients usually consumed 2 fruits daily and $35 \%$ took 1 fruit daily prior to admission. Also, $45 \%$ of the patients took 1 glass of fruit juice daily prior to admission. On admission, $55 \%$ of the patients were placed on nasogastric fluid diet while $35 \%$ were on oral fluid diet and only about $10 \%$ were on gastrostomy fluid diet. The feed method was introduced because $35 \%$ of the patients had loss of appetite while $65 \%$ of the patients had chewing or swallowing difficulty. $75 \%$ of the patients were on high protein diet fluid diet, $15 \%$ on low fat fluid diet and $5 \%$ on low protein fluid diet and Oral Hemix each. About $80 \%$ of the patients consumed between $0.5-1$ or $2.5-3.0$ litres. $90 \%$ of the patients were fed on full strength while each $10 \%$ were on half strength. Majority of the patients about $65 \%$ were fed by nurses while $35 \%$ were fed by their relatives. $40 \%$ of patients were fed 3hourly, $30 \%$ 4hourly, $20 \%$ 6hourly and $10 \% 2$ hourly. It was observed that feeding time was always adhered to by $50 \%$ of the patients while $10 \%$ never adhere to the feeding time. About $40 \%$ of the patients consumed high amount of the feed while $50 \%$ consumed low amount and only 1 patient about $10 \%$ refused to feed. $65 \%$ of the patients felt satisfied after feed intake, $15 \%$ still felt hungry and $20 \%$ just remained calm. It was noted that $10 \%$ of the patients reacted after feeding that is, vomiting-5\%, diarrhoea-5\%, whereas $90 \%$ showed no reaction to feed. All feeds were recommended by dieticians $(100 \%)$. About $40 \%$ of the patients commenced feeding two weeks after admission, $35 \%$ a week after admission, $15 \%$ more than two weeks after admission and $10 \%$ few days after admission. No patient was commenced fluid diet on the day of admission.

Table VI: Nutritional Related Problem/Diagnosis

\begin{tabular}{|l|l|l|}
\hline & f & $\mathbf{\%}$ \\
\hline Malnutrition & 2 & 10 \\
\hline Pressure ulcer & - & - \\
\hline Sepsis & 3 & 15 \\
\hline Aids & - & - \\
\hline Dysphagia & 7 & 35 \\
\hline Renal Disease & 3 & 15 \\
\hline Hepatic Disease & 1 & 5 \\
\hline Unconsciousness & 2 & 10 \\
\hline Surgery & 2 & 10 \\
\hline Total & $\mathbf{2 0}$ & $\mathbf{1 0 0}$ \\
\hline
\end{tabular}

Table VIshowed that $35 \%$ of the patients had dysphagia, $15 \%$ had renal disease another $15 \%$ had sepsis, $10 \%$ were malnourished, $10 \%$ were unconscious, $10 \%$ had surgery and $5 \%$ had hepatic disease. There was no patient with peptic ulcer or AIDS. 


\section{Discussion}

In table 1 , it was shown that majority of patients on enteral nutrition were males $(60 \%)$ whereas only $40 \%$ were females. It was also shown that the elderly of ages above 50 years $(55 \%)$ and children of ages between $0-10 y e a r s ~(15 \%)$ were mostly affected. The research which was conducted in July 2013 showed that majority of the patients were admitted in the July (50\%) and June (30\%).

\subsection{Anthropometry Measurements}

Anthropometric measurements showed that the body mass index of $35 \%$ of the patients fall within the normal range (18.5-24.9), while $60 \%$ of the patient's about $5 \%$ was overweight (25-29.9). It was also shown that $15 \%$ of the patients had $60 \%$ and below of their desirable weight while only about $5 \%$ had more than $100 \%$ of the desirable body weight. It was reported that $80 \%$ of the patients had percentage weight loss of $6-10 \%, 35 \%$ had percentage weight loss of $11-15 \%, 10 \%$ had percentage weight loss above $25 \%$. The data showed that most of the study carried out by Kelly, et al.(2000) who assigned likelihood of malnutrition scores based on serum folate and vitamin C level, anthropometric measurements, lymphocyte count, and hematocyte levels in 134 patient at a Teaching Hospital, Kelly,et al.(2000) also found that among 219 patients in an academic inner city hospital in the United Kingdom in 1998, 13\% were malnourished based on body mass index and weight loss and only these patients were referred to the Dietetic Department. In a recent British study of 2, 283 patients, malnutrition was determined by weight loss, poor intake, wounds and infections.

\subsection{Biochemical Assessment}

The biochemical assessment which is an indicator for assessing the level of depletion of important nutrients or the accumulation of toxic wastes in the body showed that $75 \%$ of the patients' haemoglobin level greater than $10.0 \times 10^{9} / 1$. The fasting blood sugar indicated hyperglycemia in $25 \%$ of the patients while $50 \%$ about half the number of the patients had normal fasting blood sugar. The fasting blood sugar for $25 \%$ of the patients was recorded. About $55 \%$ of the patients had adequate total cholesterol less than 3.6. The low density lipoproteins were normal in $55 \%$ of the patients while each of $10 \%$ of patients had lowered and raised low density lipoproteins of less than 1.9 and greater than 3.6 respectively. Half of the patients about $50 \%$ were reported to have normal levels of very low density lipoproteins $(0.4-1.3 \mathrm{mmol} / 1)$ while $15 \%$ had more than $1.3 \mathrm{mmol} / 1$ and $10 \%$ had less than $0.4 \mathrm{mmol} / \mathrm{dl}$ of very low density lipoproteins. The triglyceride levels were normal in $35 \%$ of the patients $(0.5-1.0 \mathrm{mmol} / \mathrm{dl})$, low in $20 \%$ of the patients (greater than $1.0 \mathrm{mmol} / \mathrm{dl}$ ).

The total cholesterol, low density lipoproteins (LDL), very low density lipoproteins (VLDL) and triglyceride (TG) are indices for total fat store in the body. The indices show that about $15 \%$ of the patients had depleted fat store while about $13 \%$ of the patients had an excess of fat store in the body. The lipid profile of $25 \%$ of the population was not recorded.

\subsection{Clinical Assessment}

Clinical assessment is techniques used to detect physical signs associated with nutrient deficiency, toxicity, and imbalance or even non-nutrition conditions. The clinical assessment showed that one- quarter of the patients about $25 \%$ were pale, $15 \%$ had angular stomatitis, there was oedema(retention of fluid in the body tissues) in $40 \%$ of the patients and $35 \%$ of them were wasted(depletion of the body tissues or muscles). These signs are good indicators of malnutrition.

\subsection{Dietary Assessment}

This assessment emphasizes the patients' dietary pattern. It was shown that prior to admission, $55 \%$ of the patients ate 3 meals a day while $25 \%$ ate 2 meals daily. About $45 \%$ of the patients took one snack daily while $40 \%$ took 2 snacks. $4 \%$ of the patients ate 2 fruits a day while $35 \%$ ate 1 fruit daily. It was also reported that $45 \%$ of the patients took one glass of fruit juice daily, $40 \%$ took two glasses of fruit juice and the remaining $15 \%$ took about three glass of fruit juice daily. On fluid diet, $10 \%$ were on gastrostomy fluid diet and $35 \%$ on oral fluid diet. The feeding method was introduced when $35 \%$ of the patients showed loss of appetite for food and $65 \%$ had difficulty in chewing or swallowing solid food. These conditions are among the criteria for which enteral nutrition can be administered. Carr, et al. (1996) opined that enteral nutrition is the preferred route for nutritional support; the gut barrier and immune functions are preserved and systemic infections and other complications are reduced. It was reported that $75 \%$ of the patients were on a high protein fluid diet, and $15 \%$ on low fat fluid diet. Majority of the patients ( $80 \%$ ) were fed between 1.5 to 2 litres of feed daily and $90 \%$ of the patients were on a full strength feed. $65 \%$ of the patients were fed by the nurses while $35 \%$ were fed by their relatives. The feeding time interval was 3 hourly for $40 \%$ of the patients, 4 hourly for $30 \%$ of the patients, 6 hourly for $20 \%$ of the patients and 2 hourly for $10 \%$ of the patients. $50 \%$ of the patients reported that feeding time were always adhered to; $25 \%$ were often adhered to; $15 \%$ were seldomly complied with; $10 \%$ reported that it was never adhered to; and $10 \%$ reported non-compliance. Non-compliance to feeding reported was largely due 
to negligence on the part of the nurses or patients' refusal to eat. According to Saeed,et al.(2005), one potential cause of worsening nutritional status may be inadequate medical staff awareness about the importance of nutrition in hospitalized patient. $40 \%$ of the patients consumed high amount of the feed, $50 \%$ consumed low amount of fed while $10 \%$ of the patients refused the feed. This is in support of Kondrup, et al. (2002) who stated that patients' appetite usually decreases during illness due to pain, nausea, weakness and altered mood or mental status, and they can become dissatisfied with repetitive menu cycles, dietary restriction, and the food which may not be the type prefer. It can be seen from this study that $65 \%$ of the patients felt satisfied after feeding, 155 still felt hungry while $20 \%$ just remained calm. All the feeds were recommended by a Dietician. No patient commenced feeding on the days of admission, $10 \%$ of the patients commenced feeding few days after admission, 405 commenced feeding two weeks after admission and $15 \%$ of the patients commenced feeding more than two weeks after admission. The delay in commencing feeding on patients was most times due to physical insensitivity to patients' nutritional status and delay in referring patients to a Dietician. Deborah (2005) revealed that lack of attention to functional impairment by physicians to patients upon admission and adverse drug effects are the most common causes of Iatrogenesis. Kritina (2008) opined that the nutritional status of patients is known to worsen during hospital stay which is partly due to poor recognition by the medical staff and adverse clinical routine.

\subsection{Nutritional Related Problem/Diagnosis}

According to the American Society for Parenteral and Lateral Nutrition (ASPEN, 2002), several disease states and acute events can predispose patients to malnutrition, the degree of which is usually determined by the severity of the illness. The study showed that the nutritional related problems/diagnosis for which patients were placed on enteral Nutrition were, malnutrition $10 \%$, sepsis- $15 \%$, dysphagia(inability to swallow)- $35 \%$, Renal disease- $15 \%$, hepatic (liver)disease- $5 \%$, unconsciousness- $10 \%$ and surgery-(10\%). Majority of the cases where dysphagia. This supports ASPEN (2002) that the most obvious disease states that predispose patients to malnutrition are those that prevent oral food intake such as cancer, tumours or strictures in the oesophagus, stroke and degenerative neurologic disorders that result in dysphagia.

\section{Conclusion}

In conclusion, iatrogenic malnutrition in patients was found majorly in males. The elderly and children were the most susceptible. Patients on enteral nutrition seem to be very susceptible to iatrogenic malnutrition due to factors such as non-compliance to feeding time due to negligence on the part of the nurses or patients resistance to feeding, lack of attention to functional impairment by physicians to patients upon admission, nutritional problems such as dysphagia, renal disease, sepsis, hepatic diseases, surgery and certain intrinsic factors which may result in nutrient imbalance hence causing malnutrition. Delay on the part of physicians in referring patients to the dietetics department when they can no longer manage the situation.

In extreme cases, iatrogenic malnutrition can have severe effects in the patients which include, pallor, anemia, edema, angular stomatitis, muscle wasting, nutrient imbalance, worsened health condition, susceptibility to diseases, complication, susceptibility to diseases, and complications even death. According to the British Dietetics Association (2006), malnutrition in hospital causes a reduction in muscle and physiological function which has adverse effects on health. Muscle wasting and weakness decrease mobility and stamina and impair functions of the lungs and heart. Malnutrition is also associated with poor wound healing, impaired immune incidence of post-operative complications, dehydration and presence of ulcers. The circulatory blood volume and the concentration of the serum proteins, haemoglobin and electrolytes may be reduced (Srilakshmi, 2011).

\section{Recommendations}

The following recommendations should be put in place in order to address iatrogenic malnutrition especially in patients on enteral nutrition:

1. Early detection of malnutrition by routine screening of vulnerable groups (those with chronic diseases, the elderly and children) can do much to identify those who would benefit from dietary support measures and nutrition intervention.

2. In case of patients who can eat orally, between meal snacks and liquid nutrients supplements can be helpful.

3. Emphasis should be based on frequent dense energy meals like, HEMIX and resolution of underlying causes of malnutrition, poverty, infection and illness.

4. Patients who cannot or will not eat or who are unable to absorb nutrients taken by mouth may be fed intravenously.

5. On time referral of patients by physicians to the Dietetics department would help to ameliorate the effects of iatrogenic malnutrition. 
6. It is important that clinicians initiate feeding at low calorie levels while monitoring serum electrolytes, this is because an intracellular shift of potassium, magnesium levels, thus clinicians should monitor at least daily with the onset of feeding and replete electrolytes when necessary.

7. Avoidance of excessive fluid administration is also important especially in patients with cachexia (severely malnourished).

8. Provision of multivitamin or single nutrient supplements or at least thiamine repletion may be necessary.

9. Finally, nutrition education and training of health care professionals should be advocated as this would create awareness in the minds of those professionals thereby enhancing compliance, to nutritional procedures and reducing the incidence to nutritional procedures and reducing the incidence to iatrogenic malnutrition.

10. Obsolete practices relating to nutritional screening assessment and support should be discarded and current practices should be implemented by dieticians and interventions should be timely.

11. According to the American Society for Parenteral and Enteral Nutrition (ASPEN) guidelines (2002), patients who are either unable or expected to be unable to take sufficient oral nutrition for seven to fourteen days are candidates for nutrition support. Enteral nutrition should be used if the gastrointestinal tract is functional and Parenteral nutrition should be used only if the gastrointestinal tract is not functioning or cannot be assessed (Grover et al, 2008)

\section{References}

[1]. American Society of Parental and Enteral Nutrition, (ASPEN).(2002). Guidelines for use of Parenteral and Enteral Nutrition in Adult and Pediatric Patients. Journal of Parenteral and Enteral Nutrition, 26: 18A-1385A.

[2]. American Society of Parenteral and Enteral Nutrition, (ASPEN).(2007). Nutrition Support Core Curriculum.A Case-Based Approach.The Adult Patient, Silver Spring.Md: American Society for Parenteral and Enteral Nutrition.

[3]. Antia, F.P.\&nAbraham, P. (2002).Clinical Dietetics and Nutrition ( $4^{\text {th }}$ edition). Pp 433-434

[4]. British Dietetic Association (2006).Malnutrition in Hospitals. Journal of the British Dietetic AssociationBritish Medical Association Illustrated Medical Dictionary. (2008). P.303

[5]. Churchill Livingstone Pocket Medical Dictionary. (1999) pp. 96-165

[6]. Correia, M. (2001). Risk Factors for Malnutrition in Patients undergoing Gastroenterological and Hernia Surgery: An Analysis of 374 Patients, Nutrition Hospital, 16: 5-9

[7]. $\quad$ Correia, M.I.T.B\& Campos, A.C. (2003): Prevalence of Hospital Malnutrition in Latin America

[8]. Cunningham-Rundles, S. (2005): Mechanism of Nutrient Modulation of the Immune Response; 115: pp.1-19

[9]. Deborah, C.F.(2005): MSN APRN BC: Iatrogenesis

[10]. Drewnowski, A \& Warren, M.V.A. (2001): Does Aging Change Nutrition Requirement. Journal of Nutrition Health and Aging, 5: $70-74$

[11]. Escott-Stump, S. (2008): Nutrition and Diagnosis Related Care.

[12]. Grover, A. Khashu, Mukherjee, A, Kairamkonda, V. (2008): Iatrogenic Malnutrition in Neonatal Intensive Care Units: Urgent Need to Modify Practice. Journal on Parenteral and Enteral Nutrition, 32(2): 140-144

[13]. Jacobs, J. P; Benavidez, O. J.; Bacha, E. A.; WaltersH. L. \& Jacobs, M. L. (2008). The nomenclature of safety and quality of care for patients with congenital cardiac disease: a report of the Society of Thoracic Surgeons Congenital Database Taskforce Subcommittee on Patient Safety. Cardiol Young. 18 Suppl 2:81-91 doi: 10.1017/S1047951108003041.

[14]. Kelly, I.E. Tesssier, S. \& Cahill, A. (2000): Still Hungry in the Hospital: Identifying Malnutrition in Acute Hospital Admission. QJ med 9, 93-98.

[15]. Kondrup, J, Johansen, N, and Plum, L.N. (2002): Incidence of Nutritional Risk and causes of inadequate nutritional care in hospitals. Clinical Nutrition 21(6): 461-468

[16]. Kritina, N; Claude, P; Herbert, L; \& Mathias, P. (2008): Malnutrition in Hospitalized patients. Department of Clinical Nutrition, Geneva University Hospital, Geneva, Switzerland. Vol. 27, issue 1, pp. 5-15.

[17]. Krystofiak, R.\&Mueller, C. (2007): Nutrition Screening and Assessment in the ASPEN Nutrition Support Core Curriculum: A casebased Approach. The Adult Patient, Silver Spring Md: American Society for Parenteral and Enteral Nutrition.

[18]. Singh, A, Stanza, Z \& Field J (2007): The effects of Nutrition Management on the food of malnourished patients. Clinical Nutrition, 26: 379-382.

\section{Acknowledgement}

We hereby acknowledge the contributions and support of Charles NwashiaChibuezeof the Department of Nutrition and Dietetics, University of Nigeria Teaching Hospital, Ituku-Ozalla, Enugu State,Nigeria to successful completion of this study 